Introduction: Treatment of unresectable liver metastases (LM) from uveal melanoma (UM) remains a major clinical challenge. Systemic chemotherapy and chemoimmunotherapy regimens extrapolated from cutaneous melanoma are considered to be ineffective in therapy of metastases from uveal melanoma. Studies suggest that the progression of hepatic metastases rather than the primary tumor or metastases in other organs determines survival.

Case report: We report a case of transarterial chemoembolization of 57-year-old man diagnosed with unresectable liver metastases from uveal melanoma with irinotecan eluting beads. Therapy resulted in long progression free survival and overall survival, 41 months and 45 months after diagnosis of metastatic disease respectively. Patient did not experience any major side effects of the therapy. Follow-up CTs indicate stable disease in mRECIST criteria and partial response in $\mathrm{CHOI}$ criteria.

Conclusions: Transarterial chemoembolization with drug eluting beads loaded with irinotecan may be an effective treatment of unresectable liver metastases from uveal melanoma.

Key words: endovascular procedures, melanoma, therapeutic chemoembolization, liver neoplasms, eye neoplasms/pathology.

Contemp Oncol (Pozn) 2017; 21 (3): 244-248 DOI: https://doi.org/10.5114/wo.2017.70115

\section{Long-term survival in a patient with unresectable liver metastases from uveal melanoma treated with transarterial chemoembolization with irinotecan eluting beads - case report and review of literature}

\author{
Katarzyna Stanisławska ${ }^{1}$, Honorata Stadnik ${ }^{2}$, Mikołaj Nawrocki ${ }^{1}$, \\ Katarzyna Ramlau-Piątek ${ }^{1}$, Robert Juszkat ${ }^{1}$, Michat Drews ${ }^{2}$
}

\author{
1Department of General and Interventional Radiology, Poznan University of Medical \\ Sciences, Poland \\ 2Department of General, Gastroenterological and Endocrinological Surgery, Poznan \\ University of Medical Sciences, Poland
}

\section{Introduction}

Treatment of unresectable liver metastases (LM) from uveal melanoma (UM) remains a major clinical challenge. UM is the most common primary ocular tumor in adults and the second most common melanoma after cutaneous sources, representing approximately $5 \%$ of all melanoma diagnoses. The incidence of uveal melanoma in Europe differs according to the country of origin of the patient, with an average incidence of 8 per million in the northern countries and 2 per million in the southern countries [1]. Approximately $4 \%$ of patients have metastatic disease at the time of diagnosis of the primary lesion [2]. Despite undergoing treatment of the primary tumor with either enucleation or radiation therapy, approximately $50 \%$ of patients develop metastatic uveal melanoma (MUM). The liver is the most common site of metastasis in ocular melanoma and its occurrence is estimated at 89-93\% of the cases according to different studies [2, 3]. Systemic chemotherapy and chemoimmunotherapy regimens extrapolated from cutaneous melanoma are considered to be ineffective in therapy of metastases from uveal melanoma [3]. Those with metastatic disease have poor prognosis, with median overall survival 2 to 15 months [3]. There are various possibilities of treatment of metastatic uveal melanoma, including systemic treatment with immunotherapy, chemotherapy, surgery and regional approaches such as percutaneous hepatic perfusion, hepatic isolated perfusion, radiofrequency ablation and transarterial chemoembolization (TACE) [4]. Studies suggest that the progression of hepatic metastases rather than the primary tumor or metastases in other organs determines survival [5, 6]. The present study describes a patient treated with TACE who underwent 16 TACE procedures with irinotecan eluting beads (DEBIRI), had long progression free and overall survival and did not experience any major side effects of the therapy.

\section{Case study}

The patient, a 57-year-old caucasian male, was diagnosed with left sided uveal melanoma in December 2010. The primary tumor was treated with brachytherapy Ru106. In November 2012 liver metastases were diagnosed. The patient underwent systemic chemotherapy. Subsequently, based on CT scans, as well as a clinical evaluation the patient was qualified for transar- 


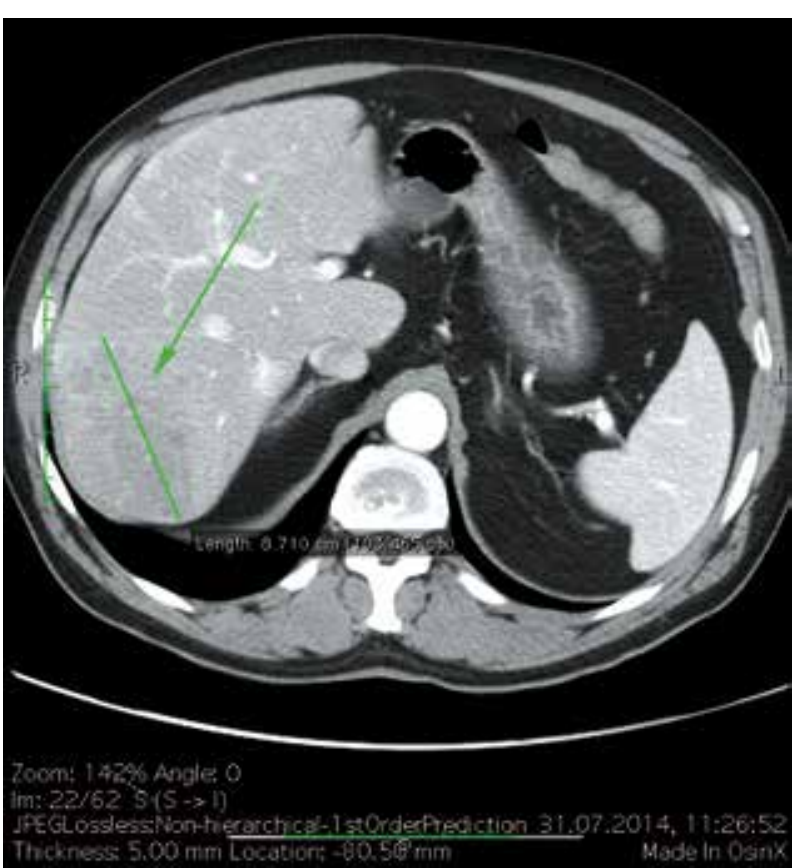

Fig. 1. Pretreatment CT shows one target lesion measuring $87 \mathrm{~mm}$ in diameter and attenuation of $81 \mathrm{HU}$ (arrow)

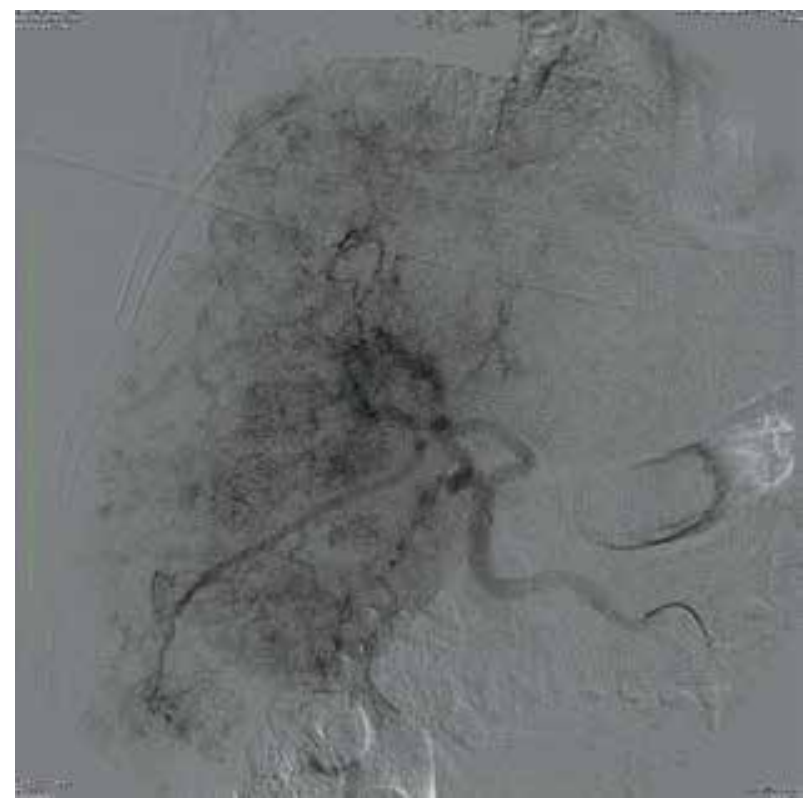

Fig. 3. Pretreatment angiogram shows diffuse pattern of contrast enhancement throughout liver without distinct nodules

terial chemoembolization (Fig. 1, 2). The liver was a dominant place of metastases, involved in less than $60 \%$ of volume and the lesions were unresectable. The angiographic image of metastases was diffuse infliltrative staining pattern without distinct nodularity (Fig. 3). He underwent four cycles of TACE between $31^{\text {st }}$ Aug 2014 and $5^{\text {th }}$ Nov 2015, 4 procedure per cycle. The procedure consisted of two stages: digital subtraction angiography (DSA) and subsequent administration of drug-loaded beads. Seldinger technique was used to access the common femoral artery, using 5F introductor (Balton, Warsaw, Poland). Superior mesenteric

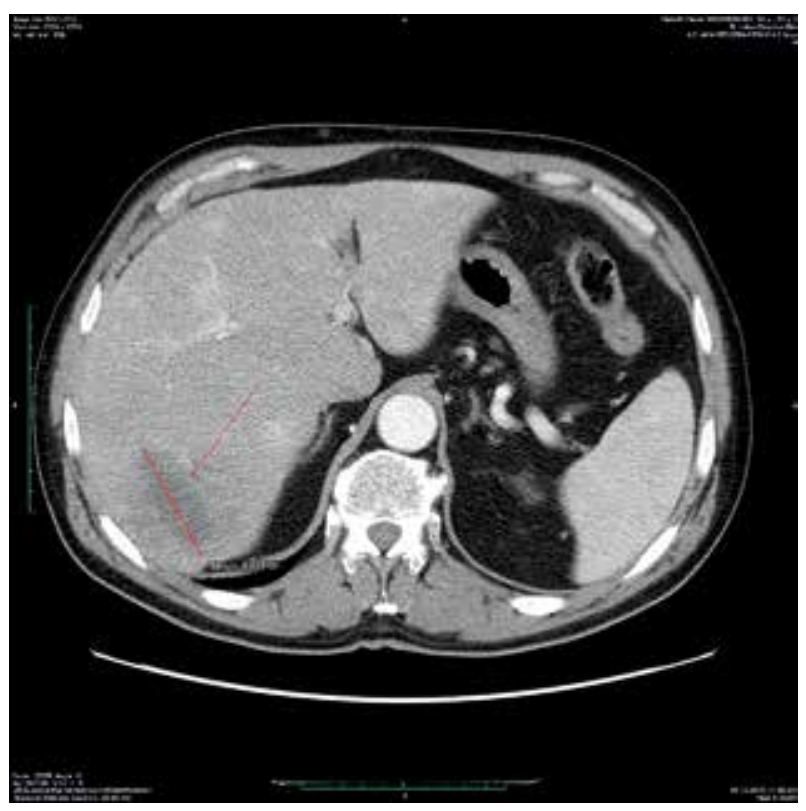

Fig. 2. CT scan after the last cycle of TACE showing decrease in diameter of the target lesion $(60 \mathrm{~mm})$ and a decrease in attenuation $-39 \mathrm{HU}$ (arrow)

and celiac trunk angiographies was performed with catheter Cobra 5F (Glidecath, Terumo, USA) to evaluate for flow direction and variant arterial anatomical features. Angiography revealed multiple, diffuse metastatic lesions in both lobes of the liver. Celiac trunk angiography was followed by subselection of left of right hepatic artery with a catheter Cobra 4F (Glidecath, Terumo, USA) and administration of $1 \mathrm{mg}$ of atropine. Subsequently, DC Beads (Biocompatibles International plc, Surrey, UK) with diameter of 100-300 $\mu \mathrm{m}$ loaded with $100 \mathrm{mg}$ of irinotecan admixed with $8 \mathrm{ml}$ of contrast medium (Ultravist 300) were infused at the rate of $1 \mathrm{ml} / \mathrm{min}$ under fluoroscopic guidance. TACE was performed under analogosedation. After each procedure patient was hospitalized on the surgical ward, usually for two days for monitoring and pain control. Treatment related morbidity was low grade and included transient loss of appetite and abdominal pain lasting for approximately 12 hours after the procedure. No major adverse reactions were noted and the patient's liver function tests and bilirubin levels were within normal limits. The procedures were performed in two week intervals.

Each TACE cycle was followed by a CT scan in order to assess the efficacy of the treatment and to qualify patient for the next cycle. Response to the treatment was quantified using modified response evaluation criteria in solid tumors (mRECIST) and modified Choi criteria. The sum of diameters of the viable target lesions was reduced by $25 \%$ after the last cycle of TACE which qualifies as stable disease in MRECIST criteria. Mean tumors attenuation of the target lesions decreased by 52\% (from $95 \mathrm{HU}$ to $42 \mathrm{HU}$ ) which combined with the decrease in the size of the tumors is a partial response according to Choi criteria.

The patient's quality of life was monitored by means of WHOQOL - BREF questionnaire before the first cycle and 
throughout the treatment. The results indicate maintenance of the overall quality of life.

In the first half of 2016, patient developed anemia which required multiple transfusions. In April 2016 PET scan indicated progressive disease with multiple metastases in peritoneum, bones and subcutaneous tissue of abdomen, head and neck. Patient died in August 2016. Therefore, the progression free survival after the diagnosis of the metastases was 41 months and 20 months after the beginning of TACE. His overall survival was 45 months.

\section{Discussion}

Although UM is the most common primary ocular malignancy in adults and uveal tract is the second most common site of melanomas, it is a rare disease with an average incidence of 8 per million in the northern European countries and 2 per million in the southern European countries [1]. Local therapy of uveal melanoma has improved significantly with increased use of conservative treatment and preservation of the eye. Nevertheless, improvements in the management of primary lesion have not been reflected in a significant increase in survival rates, and metastatic disease remains a leading cause of death among patients with uveal melanoma [7]. Up to $50 \%$ of patients develop metastatic disease and liver is most common site of metastases (89-93\%), with other common sites being the lungs (24\%) and bones (16\%) [2, 3]. The prognosis of patients with metastatic uveal melanoma is poor, with median overall survival of 2 to 15 months $[3,8]$. Studies suggest that the progression of hepatic metastases determines patients' survival more than the primary tumor or metastases in other organs $[1,5,6]$.

There are multiple possible approaches to the management of metastatic uveal melanoma, which include systemic treatment with chemotherapy, immunotherapy, surgery and locoregional approaches such as percutaneous hepatic perfusion, hepatic isolated perfusion, radiofrequency ablation and transarterial chemoembolization (TACE) $[2,3,7,9]$.

The review by Agarwala et al. analyzed the results of six comparative studies evaluating the efficacy of surgical excision as treatment of LM from UM. The matastesectomy offered a survival benefit with median overall survival ranging from 14 to 24 months after resection while survival in nonsurgical care group was 3 to 12 months. However, most of the patients with LM from UM are usually not surgical candidates and only $2 \%$ to $7 \%$ are offered surgery $[2,10]$. For the rest of the patients the possible treatment options are systemic and regional therapies. Multiple chemotherapeutic and immunotherapeutic agents have been tested in patients with MUM, however the reported response rates were generally less than $5 \%$ and the median overall survival ranged from 6.0 to 14.0 months $[2,7,9]$. Moreover, the biology of uveal melanoma differs significantly from that of cutaneous melanoma. BRAF, RAS and KIT mutations are generally rare in ocular melanoma and therefore the targeted therapies developed for cutaneous melanoma are unlikely to be efficacious in treatment of UM $[9,11,12]$. Studies of several UM specific target agents (e.g. selumatinib, sorafenib, cixutumumab, vorinostat, everolimus, pasireotide, AEB071, genetespib, cabozantinib) are being investigated [9].

As there has been no effective systemic treatment for metastatic disease and most of patients with MUM are not suitable for surgical excision, regional therapies have been investigated. They share an advantage of delivering treatment directly to radiologically evident tumors while simultaneously minimizing systemic toxicity $[2,7,13]$. The regional treatments include hepatic intra-arterial infusions (HIA), chemoembolization (TACE), and isolated and percutaneous hepatic perfusions (IHP and PHP). Some of the procedures require open surgical techniques while others are minimal access procedures. The latest studies regarding HIA with fotemustine in MUM had overall response rate ranging from 16 to $36 \%$, and median overall survival from 9 to 21 months [14-17]. In IHP the vaculature supply to the liver is surgically isolated. Melphalan has been the most commonly employed chemotherapeutic agent in that approach. A couple of studies reported high overall response rate in the range of 33 to $60 \%$, however median overall survival was between 10 and 12 months [18-19]. PHP is a nonoperative alternative to IHP. A randomized controlled phase 3 trial of PHP with melphalan compared to best alternative care reported. a prolonged hepatic progression free survial with PHP (8.1 vs. 1.6 months) with no advantage in terms of overall survival (11.4 vs. 9.9 months) [20]. A recent multi-centre study of percutaneous isolated hepatic perfusion with Melphalan conducted by Vogl et al. [21] reported median OS of 9.6 months (range 1.6-41.0 months) and median PFS of 12.4 months (range 0.9-41.0 months). The partial response rate in this study was $44 \%$ $(8 / 18)$.

Our case study, as well as reports by others demonstrate the efficacy of TACE in patients suffering from this condition. Our patient's survival of 45 months after diagnosis of metastatic disease and 24 months survival since the first chemoembolization compares favorably to other studies. Moreover, absence of any significant adverse effect despite undergoing 16 TACE procedures and stable quality of life score affirm TACE's role as a palliation. Schuster et al. [21] conducted a study of 25 patients who failed systemic therapy and were treated with TACE with fotemustine or cisplatin mixed with starch microspheres (Spherex). He reported median overall survival time of 6 months and median progression free survival was 3 months. Carling et al. [22] treated 14 patients with DEBIRI TACE and the median overall survival in this study was 9,4 months. They suggested that there was no significantly different impact on survival to other chemoembolization techniques. Venturini et al. [23] reported DEBIRI as a first-line approach in five chemotherapy-naïve patient suffering from uveal melanoma metastasis to the liver. The mean follow-up period in this study was 16,3 months. In 2005 Patel et al. [24] conducted a phase II clinical trial for patients with LMs from UM and showed that survival in patients with LMs from UM depends on the radiologic response to the treatment. Patients with complete or partial response had median survival of 21.9 months, for patients with stable disease 8.7 months and for patients with progressive dis- 
ease 3.3 months. In our case study patient had a long survival despite stable disease according to mRECIST criteria. Similar results were published by Huppert et al. in 2008 [25]. However, there are several papers based on results of treatment of hepatocellular carcinoma, that have reported necrosis of liver tumors despite absence of a substantial regression in radiologic studies [26, 27]. These can account for our patient's long survival despite having stable disease according to mRECIST. Moreover, in modified Choi criteria the patient's response was assessed as partial response. In 2008 Sharma et al. [28] reported a significantly better survival in patients with nodular angiographic pattern of liver metastases compared to patients with diffuse tumors (mean 621 days vs. 115 days). On the contrary, our patient had a diffuse angiographic pattern of liver tumors and experienced a long survival.

\section{Conclusions}

Therapy of unresectable liver metastases from uveal melanoma remains a major clinical problem. In the absence of effective systemic agents, regional therapies are an important option for patients with unresectable liver metastases from uveal melanoma. We present a case of a 56 man who was treated for LMs from UM with TACE with irinotecan eluting beads who achieved PFS of 41 months and OS of 45 months. According to mRECIST the response was stable disease while regarding modified Choi criteria it was a partial response. Moreover, absence of any significant adverse effect despite undergoing 16 TACE procedures and stable quality of life score affirm TACE's role as a palliation.

\section{The authors declare no conflict of interest.}

\section{References}

1. Jovanovic P, Mihajlovic M, Djordjevic-Jocic1 J, Vlajkovic S, Cekic S, Stefanovic V. Ocular melanoma: an overview of the current status. Int J Clin Exp Pathol 2013; 6: 1230-44.

2. Agarwala SS, Eggermont AMM, O'Day S, Zager JS. Metastatic Melanoma to the liver: a contemporary and comprehensive review of surgical, systemic, and regional therapeutic options. Cancer 2014; 120: 781-9.

3. Goh AYL, Layton CJ. Evolving systemic targeted therapy strategies in uveal melanoma and implications for ophthalmic management: a review. Clin Experiment Ophthalmol 2016; 44: 509-19.

4. Valpione S, Aliberti C, Parrozzani R, Bazzi M, Pigozzo J, Midena E, Pilati P, Campana LG, Chiarion-Sileni V. A retrospective analysis of 141 patients with liver metastases from uveal melanoma: a two-cohort study comparing transarterial chemoembolizan. Melanoma Res 2015; 164-8.

5. Diener-West M, Hawkins BS, Markowitz JA, Schachat AP. A review of mortality from choroidal melanoma. Part II. A meta-analysis of 5-year mortality rates following enucleation, 1966 through 1988. Arch Ophtalmol 1992; 110: 245-50.

6. Rajpal S, Moore S, Karakousis CP. Survival in metastatic ocular melanoma. Cancer 1983; 334-36.

7. Singh AD, Turell ME, Topham AK. Uveal melanoma: trends in incidence, treatment, and survival. Ophtalmology 2011; 118: 1881-5.

8. Sato T, Babazono A, Shields JA, Shields CL, De Potter P, Mastrangelo MJ. Time to systemic metastases in patients with posterior uveal melanoma. Cancer Invest 1997; 15: 98-105.
9. Buder K, Gesierich A, Gelbrich G, Goebeler M. Systemic treatment of metastatic uveal melanoma: review of literature and future perspectives. Cancer Medicine 2013; 2: 674-86.

10. Mariani P, Piperno-Neumann S, Servois V, et al. Surgical management of liver metastases from uveal melanoma: 16 years' experience at the Institut Curie. Eur J Surg Oncol 2009; 35: 1192-7.

11. Hofmann UB, Kauczok-Vetter CS, Houben R, Becker JC. Overexpression of the KIT/SCF in uveal melanoma does not translate into clinical efficacy of imatinib mesylate. Clin Cancer Res 2009; 15: 324-9.

12. Rimoldi D, Salvi S, Lienard D, et al. Lack of BRAF mutations in uveal melanoma. Cancer Res 2003; 63: 5712-5.

13. Lewandowski R, Geschwind JF, Liapi E, Salem R. Transcathether Intraarterial Therapies: Rationale and Overview. Radiology 2011; 259: 641-57.

14. JM, Agarwala SS and Kirkwood. Phase I/II randomized trial of intrahepatic arterial infusion chemotherapy with cisplatin and chemoembolization with cisplatin and polyvinyl sponge inpatients with ocular melanoma metastatic to the liver. Melanoma 2004; 14: 217-22.

15. Farolfi A, Ridolfi L, Guidoboni M, et al. Liver metastases from melanoma hepatic intra-arterial chemotherapy. A retrospective study. J Chemother 2011; 23: 300-5.

16. Heusner TA, Antoch G, Wittkowski-Sterczewski A, et al. Transarterial hepatic chemoperfusion of uveal melanoma metastases: survival and response to treatment. Rofo 2011; 183: 1151-60.

17. Peters S, Voelter V, Zografos L, et al. Intra-arterial hepatic fotemustine for the treatment of liver metastases from uveal melanoma: experience in 101 patients. Ann Oncol 2006; 17: 578-83.

18. Alexander HR, Libutti SK, Pingpank JF, et al. Hyperthermic isolated hepatic perfusion using melphalan for patients with ocular melanoma. Clin Cancer Res 2003; 9: 6343-9.

19. van lersel LB, Hoekman EJ, Gelderblom H, et al. Isolated hepatic perfusion with $200 \mathrm{mg}$ melphalan for advanced noncolorectal liver metastases. Ann Surg Oncol 2008; 15: 1891-988.

20. Pingpank JF, Hughes M, Alexander HR, et al. Percutaneous hepatic perfusion (PHP) vs. best alternative care for patients with melanoma liver metastases: efficacy update of the phase 3 trial. Eur J Cancer 2011; 47: abstract 9304.

21. Vogl TJ, Koch SA, Lotz G, et al. Percutaneous Isolated Hepatic Perfusion as a Treatment for Isolated Hepatic Metastases of Uveal Melanoma: Patient Outcome and Safety in a Multi-centre Study. Cardiovasc Intervent Radiol 2017; 40: 864, doi:10.1007/s00270017-1588-2.

22. Schuster R, Lindner M, Wacker F, Krössin M, Bechrakis N, Foerster MH, Thiel E, Keilholz U, Schmittel A. Transarterial chemoembolization of liver metastases from uveal melanoma after failure of systemic therapy: toxicity and outcome. Melanoma Research 2010; 20: 191-6.

23. Carling U, Dorenberg E, Haugyik S, Eide N, Bertzen, Bjorn E, Dueland S. Transarterial Chemoembolization of Liver Metastases from Uveal Melanoma Using Irinotecan-Loaded Beads: Treatment Response and Complications. Cardiovasc Intervent Radiol. 38, 2015; 38: 1532-1541.

24. Venturini M, Pilla L, Agostini G, Cappio S, Losio C, Orsi M, Ratti F, Aldrighetti L, De Cobelli F, Del Maschio A. Transarterial Chemoembolization with Drug-eluting Beads Preloaded with Irinotecan as a First-Line Approach in Uveal Melanoma Liver Metastases: Tumor Response and Predictive Value of Diffusion- weighted MR Imaging in Five Patients. J Vasc Interv Radiol 2012; 23: 937-41.

25. Patel K, Sullivan K, Berd D, Mastrangelo MJ, Shields CL, Shields JA, Sato T. Chemoembolization of the hepatic artery with BCNU for metastatic uveal melanoma: results of a phase II study. Melanoma Research 2005; 15: 297-304.

26. Huppert P E, Fierlbeckc G, Pereirab P, et al. Transarterial chemoembolization of liver metastases in patients with uveal melanoma. Eur J Radiol 2010; 74: 38-44.

27. Ebied OM, Federle MP, Carr BI, Pealer KM, LiW, Amesur N, et al. Evaluation of responses to chemoembolization in patients with unresectable hepatocellular carcinoma. Cancer 2003; 97: 1042-50. 28. Takayasu K, Arii S, Matsuo N, Yoshikawa M, Ryu M, Takasaki K, et al. Comparison of $\mathrm{CT}$ findings with resected specimens after 
chemoembolization with iodized oil for hepatocellular carcinoma.

Am J Roentgenol 2000; 175: 699-704.

29. Sharma KV, Gould JE, Harbour JW, Linette GP, Pilgram TK, Dayani PN,

Brown DB. Hepatic arterial chemoembolization for management of metastatic melanoma. AJR Am J Roentgenol 2008; 190: 99-104.

\section{Address for correspondence}

\section{Mikołaj Nawrocki}

Department of General and Interventional Radiology

Poznan University of Medical Sciences

Polna 19

60-535 Poznań, Poland

e-mail: mikolaj.nawrocki@gmail.com

Submitted: 11.05 .2017

Accepted: $\quad 9.07 .2017$ 